\title{
Management et Diversité : lignes de tension et perspectives
}

Jean-François Chanlat et Stéphanie Dameron ${ }^{1}$

\begin{abstract}
"C'est par la diversification des « rencontres», par l'épreuve des mises en relation fréquemment renouvelées, que se saisit et se vérifie ce qui est la nature même du social : son mouvement de création, de production continue .» (Georges
\end{abstract}

Balandier, 2003, p33)

Dans la plupart des pays industrialisés, la diversité est un mot en ce moment fort à la mode. La presse s'en fait largement écho, l'élection du sénateur Barack Obama comme Président des Etats-Unis en étant devenu un symbole international. Or, l'engouement pour un mot est toujours en lien avec des questions qu'une société se pose à un moment donné de son histoire. Si la nôtre ne fait pas exception à la règle, il nous faut rappeler d'entrée que la question de la diversité n'est pas en soi nouvelle et que l'intérêt de l'univers gestionnaire à son égard, non plus. En effet, d'un point de vue anthropologique, la diversité renvoie à deux problèmes fondamentaux que tout groupe humain rencontre: 1) la question du rapport à l'autre (l'altérité) et 2) la question du vivre ensemble (la socialité).

Depuis que le management existe en tant que corps de principes, cette question de la coopération entre les différentes composantes sociales d'une organisation a été posée. Et la question du comment faire en sorte que des gens différents par l'âge, le sexe, l'origine sociale, l'origine ethnique, la culture, la formation et le métier travaillent ensemble en vue de l'objectif commun a été dévolu aux gestionnaires. Tel est bien le problème qui se pose à toute action organisée et que les gestionnaires ont depuis toujours eu pour objectif de résoudre au quotidien. La question de la socialité est donc, elle aussi, au cœur de la dynamique managériale (Dameron, 2005).

C'est au cours des deux dernières décennies où l'esprit gestionnaire anglo-saxon s'est particulièrement diffusé dans le monde que le vocable « diversité » s'est imposé, peu à peu, dans le domaine de la gestion (Kirkon et Greene, 2004).

. Dans les sociétés développées, son usage est le fruit de la rencontre de quatre grands mouvements de fond observés depuis l'après-Guerre :

- une présence désormais massive et permanente des femmes sur le marché du travail (Méda, 2008) ;

- un brassage démographique entraîné par les divers mouvements migratoires (Héran, 2007);

- une mondialisation des échanges ;

- une culture plus tolérante et libérale à l'égard des différences ;

Et d'un certain nombre de constats observés à des degrés divers dans de nombreux pays notamment, en France (Barth et Falcoz, 2007).

- des discriminations sociales multiples d'accès à l'emploi ;

\footnotetext{
${ }^{1}$ Université Paris-Dauphine, DRM.
} 
- une division très sexuée du travail et une faible représentation féminine dans les sphères dirigeantes (Laufer, 2007) ;

- des conflits interculturels plus médiatisés (Davel, Dupuis et Chanlat, 2008) ;

- la difficulté de nombreux jeunes à s'insérer dans le marché du travail ;

- l'éviction des salariés de plus des 50 ans de la vie active ;

- les difficultés d'insertion rencontrées par les personnes handicapées.

En France, l'intérêt des cercles gestionnaire pour cette question est encore plus récent que dans les univers anglo-saxons. Mais il reste que, au cours des dernières années, nous avons assisté à une multiplicité d'actions dans ce sens, ,par exemple, : à la création de la Halde (Haute Autorité de Lutte contre les Discriminations et pour l'Egalité.), à la fondation du Club du XXI siècle et d'une association des managers de la diversité (l'AFMD) qui visent à accroître la représentativité de différents groupes peu ou mal représentés dans la vie sociale, à la création toute récente d'un Haut Commissariat à la diversité et de plusieurs chaires universitaires de recherche dédiées à ce thème, dont une à Dauphine ${ }^{2}$, à de nombreux colloques et débats concernant cette question, à un programme de labellisation des organisations (Le label diversité) et à la signature de chartes de la diversité par de nombreuses grandes entreprises (Point, 2006) .

La gestion étant un monde où l'on voit apparaître régulièrement des modes, d'aucuns peuvent considérer cet engouement actuel pour la diversité comme une autre illustration de ce phénomène. Tout comme la qualité était, par exemple, un des slogans populaires dans les entreprises des années 80, la diversité semble être devenue avec le développement durable, celui du monde managérial de ce XXI siècle naissant.

Si le débat sociétal sur la diversité trouve un écho particulier en entreprise c'est que celle-ci connaît des évolutions fondamentales. Quatre d'entre elles nécessitent une réflexion renouvelée sur le management de la diversité. L'entreprise du XXIème siècle est en effet multipolaire, les centres de décisions sont répartis dans différents territoire; elle est multiculturelle, ses employés, ses fournisseurs et ses clients sont de différentes origines; elle est multigénérationnelle - les stages et les formations en apprentissages se généralisent et commencent plus tôt tandis que l'âge de départ à la retraite recule ; elle est enfin distribuée et virtuelle, ses équipes et son encadrement travaillent à distance utilisant abondamment les technologies de l'information et de la communication à leur disposition. De ce fait, les processus actuels, notamment de recrutement, de gestion des parcours professionnels et de diffusion et partage des connaissances, sont appelés à évoluer radicalement.

L'objectif de cette communication est ainsi, après avoir présenté le caractère polysémique de la notion de diversité suivant une perspective comparée, de repérer les tensions exercées sur les organisations par le management de la diversité et de dégager les perspectives de recherche sur cette thématique.

\footnotetext{
${ }^{2}$ La chaire 'Management et diversité' de l'Université Paris-Dauphine est sous la responsabilité des Professeurs Jean-François Chanlat et Stéphanie Dameron. Elle a été fondée en partenariat avec le Club du XXI siècle et quatre entreprises : EADS, GDF-SUEZ, LE GROUPE LA POSTE et LA MACIF qui lui apportent leur soutien financier. Elle a été lancée en janvier 2009.
} 


\section{La diversité : un élément de la gestion d'entreprise}

Dans le contexte actuel, la diversité est devenue un élément de la stratégie de nombreuses grandes entreprises, notamment si l'on en juge le discours qu'elles tiennent à ce sujet. Elle est aussi perçue par certains gouvernements comme un instrument d'une politique sociétale plus large, en matière d'égalité des chances, à poursuivre dans les différentes sphères de l'économie, des medias, de la culture, de l'administration et de la politique.

Le dénominateur commun de ce management de la diversité part d'un postulat, partagé par tous les acteurs de la mise en place de telles politiques, que les différences, quelles quelle soient, peuvent représenter à la fois des ressources et un atout indispensable à la bonne marche des organisations. On peut définir ainsi le management de la diversité comme le dernier développement d'un ensemble de stratégies managériales dont le but est non seulement d'améliorer la présence et le sort, dans certains contextes professionnels, de personnes appartenant à des minorités discriminées ou exclues mais aussi de rendre l'organisation plus efficace en exploitant au mieux ces différences (Sabeg et Charlotin,2006).

Cette stratégie se distingue quelque peu des programmes d'égalité en emploi. Car, contrairement à ces derniers qui cherchent à promouvoir d'abord et avant tout une égalité des chances face à l'emploi, la gestion de la diversité vise d'abord et avant tout à améliorer la compétitivité et l'efficacité des entreprises en mettant l'accent sur la reconnaissance et l'apport des différences sociales et en mettant en place des pratiques dans ce sens. La gestion de la diversité cherche à faire du lieu de travail un endroit intégrateur pour tout le monde, et à promouvoir une culture qui accepte les différences afin de rendre l'organisation plus performante et plus efficace dans le contexte qui est le sien; autrement dit, les différences doivent faire la différence.

En anglais, la notion de "diversity » recouvre par ailleurs plusieurs acceptions. Elle peut signifier à la fois l'hétérogénéité du personnel et la gestion de la diversité de ce personnel. Elle peut également renvoyer à n'importe quelle politique concernant les immigrants ou les minorités, aux programmes d'accès à l'égalité, ou encore faire référence à toute pratique mise en place pour faire face à l'hétérogénéité sociale.

Comme le montre, par exemple, une étude récente qui a comparé 241 sites internet d'entreprises européennes sur cette question, les entreprises britanniques sont, celles qui ont le discours le plus large et le plus proactif dans ce domaine alors que les autres se contentent de valoriser essentiellement l'accès à l'emploi et d'améliorer les chances de promotion des personnes appartenant à des catégories exclues ou discriminées (femmes, minorités ethniques, seniors, handicapés, homosexuels..) (Point, 2006).

Le contexte sociohistorique jouant un grand rôle dans la construction différencié des discours, voyons maintenant comment cette question est vue dans le contexte français.

\section{Management et diversité en France : un regard comparé}

Comme nous l'avons souligné dans notre introduction, la question de la diversité et de sa gestion, prise dans son acception anglo-saxonne, est une question tout à fait récente dans le contexte français. Même si nous avons eu tout un débat il y a quelques années sur la parité dans le domaine politique, notamment entre hommes et femmes et des lois cherchant à lutter contre le racisme et les discriminations sociales, le recours au mot diversité dans notre contexte hexagonal est un fait social nouveau. Outre l'influence qu'exerce l'expérience 
anglo-saxonne à ce sujet et que nous venons d'évoquer brièvement, son usage en France a émergé à l'occasion de deux grandes questions sociales :

- les problèmes liés aux discriminations vécues par les jeunes des banlieues dites sensibles ;

- la faible représentation dans les positions de pouvoir (notamment économique et politique) de personnes issues des minorités ou ayant des profils atypiques.

Pourquoi cette question, largement discutée par exemple en Amérique du Nord depuis des années, ne s'est-elle donc pas posée de la même manière en France qu'Outre-Atlantique ? Pour une raison bien simple, c'est que la France ne définit pas tout à fait la citoyenneté de la même manière (Weil, 2005 ; Schnapper, 2007).

Depuis la Révolution française, les responsables politiques français ont toujours défendu en effet une conception universelle de la citoyenneté et n'ont jamais fait référence aux origines ou au genre. Il fallait constituer l'unité de la Nation et cette unité ne pouvait pas se fonder sur des différences communautaires de quelque nature que ce soit. Toute l'expérience politique a été de renforcer cette notion d'unité de la Nation et donc l'assimilation pleine et entière des immigrés ou des provinciaux à la culture commune (affirmation du français comme seule langue nationale, francisation de toutes les régions du pays via l'école publique obligatoire, loi sur la laïcité, défense des idéaux de la République, loi sur le voile, etc..). Comme le rappelle fort justement Pascal Combemale (2009, p77), le préambule de notre Constitution hérité de celui de 1946 en fait foi :

«Au lendemain de la victoire remportée par les peuples libres sur les régimes qui ont tenté d'asservir et de dégrader la personne humaine, le peuple français proclame à nouveau que tout être humain, sans distinction de race, de religion, ni de croyance, possède des droits inaliénables et sacrés. »

\section{Les principales lignes de tension de la gestion de la diversité}

Le management de la diversité renvoie fondamentalement à la socialisation au sein d'un groupe. Pour reprendre le sociologue E. Durkheim, la socialisation résulte à la fois de processus d'intégration et de processus de régulation. L'intégration sociale nécessite que les membres d'une même entité, et même si c'est avec une intensité inégale, partagent une identité commune, entendue comme un ensemble de valeurs, croyances et normes de comportement. L'intégration implique également que ses membres soient en interaction les uns avec les autres. Elle nécessite enfin qu'ils soient voués à des buts communs. Par ailleurs, la régulation sociale, seconde dimension de la socialisation, renvoie au pouvoir qui règle les activités des individus.

Socialisation ne veut donc pas dire acceptation aveugle des différences, relativisme absolu et laissez-faire. Bien au contraire, elle exige de définir un ensemble de référents partagés stables à partir desquels la variation des comportements est acceptable.

Dans ce cadre, trois grandes lignes de tension parcourent à des degrés divers, selon les pays, la gestion de la diversité..

La première touche la tension égalité/diversité, la seconde, la tension laïcité/diversité et la troisième, performance/diversité.

\section{La tension égalitéldiversité}

Nos sociétés démocratiques étant fondées sur l'égalité des droits, la gestion de la diversité, par certaines de ses pratiques, revisite la question de l'égalité. Ce qui suscite des débats fort nombreux, notamment en France. Depuis Tocqueville, nous savons que nous vivons dans 
des sociétés qui aspirent à l'égalité sociale. Mais nous savons aussi par Marx que les droits formels ne sont pas toujours réels. Le principe d'égalité est donc toujours un idéal à poursuivre et toute inégalité fortement ressentie peut alimenter le mouvement social. C'est donc en son nom que l'on a mis en place, comme nous l'avons vu précédemment, des politiques d' "affirmative action» aux Etats-Unis, lesquelles devaient permettre aux minorités discriminées (Noirs et femmes notamment) d'améliorer leurs chances. Mais c'est aussi au nom de l'égalité que certaines de ces pratiques associées à ce mouvement : mises en place de quotas, discrimination positive ont été particulièrement critiquées (Calvès, 2004).

En France, la loi sur la parité hommes/femmes en politique a subi le même type de critique, de la part de féministe, comme, par exemple, Elisabeth Badinter qui, dans le cas français, s'appuyait sur l'esprit républicain pour rejeter le projet de loi proposé. L'idée d'établir des 'statistiques ethniques' suscite actuellement des débats vigoureux et des critiques similaires. Car la mise en place éventuelle de tels indicateurs dans notre contexte ne va pas de soi. Elle rentre non seulement en contradiction avec la conception de la citoyenneté à la française que nous venons d'évoquer, mais aussi pose des problèmes particulièrement ardus sur le plan méthodologique et peut enfin avoir des effets redoutables sur la définition de soi. Il ne faut jamais oublier en effet que, selon la recherche dans le domaine des relations ethniques, ce sont les interactions que les groupes ont entre eux et les limites qu'ils établissent dans un contexte donné qui constituent le niveau d'analyse pertinent.

A une analyse à partir de catégorisations de type ethno-racial tendant à enfermer les personnes dans des cadres naturels et essentialistes, il faut donc préférer une analyse en termes d'ethnicisation des rapports sociaux. Les représentations d'une génération d'enfants français de parents immigrés ne sont pas en effet analogues à celles de leurs parents en raison de conditions socio-historiques différentes (taux de chômage, degré de ségrégation spatiale, maîtrise de langue, niveau d'instruction, etc..). Les rapports sociaux sont par définition dynamiques et historiquement datés. On ne peut donc les enfermer dans des catégories figées (Combemale, 2009). Une telle conception est essentielle à défendre si nous voulons maintenir notre conception républicaine de l'indifférenciation raciale et nous attarder à ce qui est fondamental : analyser les logiques qui concourent, comme nous y invite Robert Castel (2007) aux discriminations négatives et à l'exclusion sociale.

Outre la posture philosophique, propre à la conception française du vivre ensemble, que nous venons de rappeler, l'attachement des Français envers une vision égalitaire et universelle du citoyen bénéficie également par ailleurs de solides fondements historiques et anthropologiques. La notion d'égalité entre les frères et les soeurs qui est au coeur de la structure familiale dominante française, est peut-être une des explications de cette passion bien française pour l'égalité. Ce qui n'est pas le cas de la structure familiale dominante en Angleterre, laquelle accepte volontiers depuis plusieurs siècles l'inégalité entre les frères et les sœurs avec la règle de la primogéniture en matière d'héritage (Todd, 1984).

D'autres critiques, plus radicales encore, en appellent à l'égalité contre la diversité. Ils voient dans la popularité croissante de l'idée de diversité un écran de fumée qui masquerait la montée des inégalités sociales que nos sociétés ont connues au cours des trente dernières années. La présence de représentants des minorités à des postes de responsabilité ne serait pas alors synonyme d'une amélioration de l'égalité sociale mais bien le signe d'une accession de certains d'entre eux ou d'entre elles aux groupes dominants (Michaels, 2009). Cette politique, si elle ne change pas la mécanique fondamentale des inégalités, permet d'améliorer en revanche sa légitimation auprès des groupes socialement défavorisés. Les cercles du pouvoir et le système social ne seraient pas globalement plus ouverts mais en sortiraient renforcer. 
Face à de telles critiques, les défenseurs des législations et des pratiques mises en places au nom de la diversité défendent l'idée que si nous laissons les choses en l'état, il n'y aurait que des améliorations fort lentes et que les personnes concernées seraient toujours exclues de nombreux emplois et postes hiérarchiques. En d'autres termes, la parité, les quotas et la discrimination positive seraient des réponses pragmatiques à l'inégalité sociale ambiante. Les résultats obtenus par les pays scandinaves en ce qui concerne la parité hommes/femmes (Méda, 2008), et ceux obtenus aux EU dans les universités semblent leur donner en partie raison. Mais il reste que ces politiques continuent de poser des problèmes au principe d'égalité, qu'elles peuvent entrainer des effets pervers pour ceux et celles qui en sont les bénéficiaires et qu'elles rentrent parfois en contradiction avec certains principes de base de la vie commune. Ce qui nous amène au deuxième type de tension.

\section{La tension laïcité/diversité}

Une deuxième tension a en effet surgi, notamment en France, en relation avec la gestion de la diversité. C'est la tension entre diversité et laïcité. Elle a été provoquée à la fois par l'apparition de pratiques et de comportements religieux observés en milieux de travail et dans certaines institutions publiques et par les revendications de la reconnaissance de ces pratiques dans les univers professionnels. Si la question du foulard à l'école en a cristallisé dans les dernières années en France l'essence, d'autres cas moins médiatisés mobilisent régulièrement les gestionnaires et les responsables administratifs : mise en place de lieux de prière sur le lieu de travail, respect des interdits alimentaires, prise en compte des périodes de jeûne, port de signes religieux, exigence de séparation des sexes, contestation du contenu des enseignements... Ces situations que l'on retrouve également dans d'autres pays industrialisés, sont vécues de manière particulière en France car elle pose le rapport à la laïcité.

Comme chacun le sait, la France est un pays qui reconnaît la liberté de croyance mais qui a séparé dans la plus grande partie de l'Hexagone, les départements concordataires d'AlsaceMoselle faisant exception à cette règle, les confessions religieuses et l'Etat. Ce qui veut dire, que les institutions publiques sont, par définition, laïques, la laïcité garantissant l'exercice de la liberté de conscience, notamment en matière religieuse. C'est donc influencé par cet esprit républicain que la commission Stasi a été l'inspiratrice d'une loi passée en 2004 qui a interdit dans les écoles, les collèges et les lycées publics, le port de signes ou tenues par lesquels les élèves manifestent ostensiblement une appartenance religieuse.

\section{La tension performance/ diversité}

La question de la gestion de la diversité, comme nous l'avons vu, est dans une large mesure poussée par des objectifs d'efficacité et de performance. Si, selon la nature de l'organisation (privée, publique, coopérative ou associative), les finalités peuvent bien sûr variées, il reste que le bon fonctionnement demeure une exigence permanente pour le management. Plusieurs éléments de la diversité peuvent alors jouer pour ou contre ces impératifs.

En effet, la diversité culturelle n'est pas toujours un atout. Elle peut susciter de nombreux problèmes. C'est la raison pour laquelle une sous-discipline du management, le management interculturel (Barmeyer et Chanlat, 2004 ; Davel, Dupuis et Chanlat, 2008) s'est constituée, au cours des vingt dernières années, pour y faire face. La recherche met bien en évidence combien les relations interculturelles ne sont pas toujours sources d'enrichissement mais peuvent être problématiques et affectées la performance des organisations concernées (Chevrier, 2000 ; Dameron et Joffre, 2007). La mondialisation des échanges, le nombre de 
fusions et acquisitions, la cosmopolitisation de nos sociétés, les nombreuses négociations internationales et la formation d'unions régionales, notamment la construction européenne, servent de cadre à de telles réflexions.

La volonté des uns rencontre en effet toujours celle des autres. Or, chacune de ces volontés reposent la plupart du temps sur des représentations fort différenciées de la manière de vivre ensemble.

Dans le cas de la gestion de la diversité, le postulat de base est bien sûr que celle-ci est tout à fait positive pour toute organisation. Et qu'il faut donc mettre en place les conditions adéquates pour exploiter ces différences sources d'enrichissement, d'innovation et de performance. Car il est un fait également noté par la recherche que l'innovation passe par les déviants (Alter, 2004), que la diversité est essentielle dans un environnement varié, que des regards multiples sont sources de développement et qu'une organisation qui est ouverte aux différences est mieux perçu par le corps social. Pourtant, la diversité n'est pas nécessairement source de performance dans les organisations; la diversité pose question. Si celui qui est différent peut être source de richesse et d'innovation par le fait de penser autrement, ou encore d'être connecté à d'autres formes de réseaux sociaux, il a également un déficit initial de socialisation et porte en lui le risque du communautarisme et de l'enfermement.

En ce sens, les recherches sur la performance de groupes diverses présentent des résultats ambivalents. Certains travaux mettent en exergue leur capacité d'innovation et de créativité. Les travaux de Crozier et Friedberg (1977) par exemple mettent en évidence le rôle du marginal sécant, capable de connecter deux réseaux dans une organisation. De même, les recherches de Watson et alii (93) ou Distefano (2000) révèlent la richesse du processus de décision au sein de groupes diverses, et la capacité de trouver des solutions plus innovantes quand des points de vues différents peuvent être connectés.

Cependant, d'autres travaux soulèvent les difficultés de la gestion d'équipes diverses. Les théories de l'identification sociale montrent combien l'existence de différents sous groupes, différentes communautés au sein d'une même équipe, peut exacerber le clivage communautaire et renforcer le sentiment d'appartenance au sous groupe. (Hogg et Abrams, 1999; Hewstone, Rubin and Willis, 2002). L'équipe étant perçue comme une menace à l'appartenance identitaire au sous groupe, l'inclusion des différentes communautés peut générer des réactions défensives et affaiblir la capacité d'intégration au sein d'un groupe plus large (Milliken and Martins, 1996; Hornsey and Hogg, 2000; Shapiro et al., 2002; Jayne and Dipboye, 2004).

Une explication à cette ambivalence de la littérature en sciences de gestion dans la relation entre diversité et performance peut se trouver dans les travaux fondateurs de Durkheim sur la division du travail social et la mise en évidence de deux formes de cohésion sociale: la solidarité organique, fondée sur la différenciation, et la solidarité mécanique, basée sur la ressemblance (Durkheim, 1893). Cette dualité du social permet d'éclairer la nature des relations coopératives suivant un double mécanisme : la coopération complémentaire et la coopération communautaire (Dameron 2002; Dameron, 2004) La coopération complémentaire repose sur la différenciation des individus et la recherche de ressources complémentaires. Elle se développe suivant des modalités stratégiques, c'est-à-dire de calcul individuel dans la relation à autrui. Si la coopération complémentaire repose sur la différence, c'est la quête de ressemblance qui génère la coopération communautaire. En effet, cette coopération est fondée sur l'appartenance à un groupe auquel l'individu s'identifie. Elle se développe dans la construction, la protection et la défense de cette identité perçue comme 
commune. Les travaux sur l'identité sociale (Ashforth \& Mael, 1989; Hogg \& Abrams, 1999 ; Tyler, 1999) analysent les mécanismes cognitifs sous-jacents à la coopération communautaire. Suivant la forme de coopération étudiée, souvent peu explicitée dans les travaux de recherche, les avantages ou les difficultés seront mis en avant. La coopération complémentaire reposant sur la diversité des ressources, elle est favorisée par la diversité des membres. La coopération communautaire étant fondée sur le besoin d'appartenance, la diversité des membres, à travers les différentes valeurs qu'ils portent, sera perçue comme une menace à l'appartenance identitaire des différents sous groupes. Il s'agit alors de différencier l'analyse en fonction de la nature des relations coopératives étudiées,

\section{Quelles perspectives de recherches dans le domaine du management de la diversité ?}

Les travaux qui existent aux Etats-Unis pour mesurer l'efficacité des programmes et politiques en matière de diversité débouchent toutefois sur des résultats mitigés. En effet, une grande étude longitudinale américaine portant sur la période 1971-2002, menée auprès de 708 grandes entreprises privées, a montré que des trois type de pratiques étudiées, les pratiques les plus efficaces en matière de diversité étaient celles qui assignaient des responsabilités organisationnelles très précises (autorité et expertise) à travers la mise en place de programmes, d'équipes, de comité et de managers dédiés spécialement à la diversité. Ces stratégies de type organisationnel étaient efficaces pour accroître la proportion d'employés et de managers noirs (hommes et femmes) et de femmes. En revanche, les séances de formation pour combattre les préjugés et les stéréotypes, tout comme les séances individuelles de feedback, ont montré peu d'efficacité à cet égard. Dans certains cas, elles entraînaient même une réaction négative en retour. Enfin, les résultats concernant le dernier type de pratiques, mis en place pour rompre l'isolement social des femmes et des minorités discriminées via le mentorat et la mise en réseau, se sont également avérés décevants.

En Europe, la réflexion met aussi l'accent sur une stratégie organisationnelle intégrée, certains insistant sur la création d'espaces de parole adéquats d'une part, pour échanger sur les pratiques et d'autre part, pour exprimer ces propres difficultés dans la gestion au quotidien de cette diversité. La dynamique sociale au travail se fondant sur une authentique reconnaissance des apports de chacun, il est en effet essentiel de s'assurer de créer des médiations expressives dans les univers professionnels. C'est la seule façon de dire aux autres qu'ils ou elles existent et leur éviter de rester invisibles aux yeux de tous.( Corne, Warland et Pinson, 2008 ; Mutabazi et Pierre, 2008))

Dameron et Joffre (2007) montrent que les représentations des différences culturelles sont souvent mobilisées dans un sens négatif, avec effets miroirs. En mettant en évidence les facteurs modérateurs de la perception négative de la diversité et son impact dans le développement de la coopération au sein d'un groupe (Dameron et Joffre, 2007), il est possible de repérer quatre voies de recherche qui peuvent s'avérer potentiellement riches d'enseignements pour la gestion de la diversité.

Premièrement, la connaissance intuitu personae s'avère être un modérateur puissant de l'usage de stéréotypes négatifs. Pour favoriser cette connaissance interpersonnelle, une meilleure compréhension de la construction des réseaux sociaux, notamment en dynamique pour pouvoir les favoriser et les orienter, est nécessaire (Ingram et Morris, 2007). En parallèle, les recherches sur les relations affectives dans les organisations, comme l'amitié par 
exemple, restent trop rares en sciences de gestion et sont pourtant également nécessaires donner l'envie de coopérer (Alter, 2009).

Les effets structurels et de management du groupe sont également des clefs pour modérer le risque de sentiment d'injustice: les travaux sur l'identification sont en plein essor pour repérer comment favoriser l'identification à ce groupe secondaire qu'est l'entreprise, pour reprendre la classification des groupes d'Anzieu et Martin. De même, dans un univers moins hiérarchisé, plus en réseau, le leadership et le rôle du leader sont également des dimensions clefs pour favoriser et animer le sentiment d'équipe. Il s'agit de réinventer, dans cet univers organisationnel plus flou du point de vue de sa structure, où les lignes de rattachement sont multiples, des modalités de leadership qui dépassent très largement une légitimité de statut.

\section{Conclusion}

Comme nous venons de le rappeler, la question de la diversité et du management n'est pas une question récente pour les sciences du social, voire pour le management lui-même. Faire du collectif avec un ensemble varié de personnes est par définition l'objet même de la gestion. Car tout groupe humain est toujours plus ou moins diversifié en termes de sexe, de classe d'âge, de formation, de métier, d'origine sociale, et d'origine ethnique.

Si l'impératif gestionnaire vise justement, depuis l'émergence du management en tant que corps de principes institués, l'efficacité d'un collectif organisé, nous avons vu que les transformations sociales des trente dernières années ont amené des interrogations et des débats dans de nombreux pays industrialisés et notamment en France, autour de l'idée de gérer ces nouvelles différences.

Or, penser la diversité, c'est, nous venons de le voir, plusieurs choses à la fois. C'est tout d'abord comprendre les différences en rendant intelligible les cadres symboliques dans lesquels les personnes pensent (D'Iribarne,2008).. Tel est l'objet du management interculturel. Penser la diversité, c'est ensuite rendre compte des inégalités et des problèmes vécus par des personnes qui dans un contexte social donné se retrouvent discriminés, victimes de racisme, d'homophobie, voire exclus. Tel est l'objet des sciences du social s'intéressant à la diversité et en particulier à son management. Penser la diversité, c'est enfin tenter d'équilibrer au mieux les différences de chacun (groupes et ou individus) avec les principes du vivre ensemble dans une société démocratique. Tel est l'objet de la gouvernance politique. En d'autres termes, penser la diversité aujourd'hui, c'est participer à la construction d'un vivre ensemble qui tiennent compte dans chaque contexte concerné à la fois des nouvelles réalités du monde, des aspirations individuelles de chacun et des idéaux démocratiques qui nous guident.

Les organisations et les entreprises, en raison du rôle qu'elles jouent dans la dynamique socioéconomique contemporaine et les gestionnaires qui en ont la responsabilité ne peuvent pas l'ignorer. Car les pratiques managériales, en tant que pratiques sociales situées, exigent pour les comprendre une double anthropologie, une anthropologie générale qui s'intéresse à l'être humain générique et une anthropologie singulière qui s'intéresse aux personnes concrètes et en situation. Ces pratiques étant au cœur des questions débattues de nos jours autour de la notion de diversité, la réflexion managériale ne peut pas éviter de faire ce détour anthropologique. Car, au-delà de cette question, nous avons vu qu'un autre impératif d'ordre éthique et politique se dessine : construire des mondes sociaux qui respectent à la fois les 
valeurs qui nous guident et les individus et groupes concernés qui en font partie. Tel est le défi que les acteurs sociaux du monde des organisations du XXI siècle devront également surmonter. Par la même, nous remettons au premier plan, comme le réclamait déjà Marcel Mauss en 1920, l'étude et la discussion de ce qui fonde l'harmonie sociale dans ces nouveaux mondes organisés que nous sommes en train de construire où la définition culturelle de soi a pris une importance croissante.

\section{Références}

Alter, N., Donner et recevoir, la coopération dans l'entreprise, Editions La Découverte, 2009. Ashforth, B.E. and Mael, F. (1989), "Social Identity Theory and the Organization", Academy of Management Review, 14(1): 20-39.

Balandier, G, Civilisés, dit-on, Paris, PUF, 2003

Barmeyer, C et Chanlat, J-F, (coord), Cultures, nations et gestion, numéro thématique, Management international, 2004.

Barth, I et Falcoz, C, (Dir), Le management de la diversité Enjeux, fondements et pratiques, Paris, L'Harmattan, 2007.

Calvès, G, La discrimation positive, Paris, PUF, collection Que sais-je ?, 2004.

Castel, R, La discrimination négative, Paris, Seuil, 2007.

Chanlat, J-F, «Le défis social du management : l'apport des sciences sociales », in Kalika, M

(dir), Les défis du management, Paris, Editions Liaisons sociales, 2002.

Chanlat, J-F, (Dir), L'individu dans l'organisation, Les dimensions oubliées, Ste Foy, Les

Presses de l'université Laval, Paris, Eska, 1990 ; 2005.

Chanlat, J-F, Sciences sociales et management Plaidoyer pour une anthropologie générale, Ste Foy, Les Presses de l'université Laval, Paris, Eska, 1998.

Commission Européenne, Rapport annuel sur l'égalité et l'antidiscrimination : vers la diversité, rapport, Bruxelles, 2003.

Combemale, $\mathrm{P}$, « L'ethnicité, une notion aux dimensions historique et sociale », Alternatives économiques, no 280, mai 2009, pp77-79.

Cornet, A, Warland, P et Pinson, GRH et gestion de la diversité, Paris, Dunod, 2008.

Crozier, M. and Friedberg, E. (1977), L'acteur et le système, Editions du Seuil.

Dameron S. (2002), « Les deux conceptions du développement de relations coopératives dans l'organisation », in I. Dostaler \& H. Laroche (Eds.), Perspectives en management stratégique, EMS Editions.

Dameron S. (2004), « Opportunisme ou besoin d'appartenance ? La dualité coopérative dans le cas d'équipes projet»,M@n@gement, vol.7,n³, p. 137-160.

Dameron, S et Joffre, O, 'The good and the bad : the impact of cultural diversity on

cooperative relationships', International Journal of Human Resource Management, vol 18, no 11, 2007.

Davel, E, Dupuis, J-P et Chanlat, J-F, (Dir), La gestion en contexte interculturel, Ste-Foy, Les Presses de l'université Laval ; CDRom, Télé-Université du Québec, Montréal, 2008.

Distefano, J.J. and Maznevski, M.J. (2000), "Creating Value with Diverse Teams in Global Management", Organizational Dynamics, 29(1): 45-63.

Durkheim, E. (1930), De la division du travail social, PUF/ Quadrige, 1996.

Halde, Rapport annuel, Haute Autorité de Lutte contre les Discriminations et pour l'Egalité, Paris, Halde, 2007.

Héran F, Le Temps des immigrés, Paris, Seuil, 2007. 
Hewstone, M., Rubin M. and Willis H. (2002) "Intergroup bias", Annual Review of Psychology, 53(1): 575-605.

Hogg M.A. et Abrams D. (1999), Social identification : a social psychology of intergroup relations and group processes, Routledge.

Hornsey, J.H. and Hogg, M.A. (2000) "Assimilation and diversity: an integrative model of subgroup relations", Personality and Social Psychology Review, 4(2):143-156.

Ingram, P., \& Morris, M. 2007. Do People Mix at Mixers? Structure, Homophily, and the "Life of the Party. Administrative Science Quarterly, 52 (4): 558-585.

D'Iribarne, P, Penser la diversité du monde, Paris, Seuil, 2008.

Jayne, M.E. and Dipboye, R.L. (2004), "Leverage Diversity to Improve Business

Performance: Research Findings and Recommendations for Organizations", Human Resource Management, 43(4): 409-425

Kirkon, G et Greene, A-M, The Dynamics of Managing Diversity, A Critical Approach, Londres, Butterworth-Heineman, $2^{\text {nd }}$ édition, 2004.

Laufer, J, « L égalité professionnelle hommes femmes-Une mise en perspective », Chapite 1, tiré de Barth, I et Falcoz, C, (Dir), Le management de la diversité Enjeux, fondements et pratiques, Paris, L'Harmattan, 2007, pp 25-49.

Méda, D, Le temps des femmes, Paris, Champ, Flammarion, 2002 ; 2008.

Michaels, W,B, La diversité contre l'égalité, Paris, éditions Liber, 2009.

Milliken, F.J. and Martins, L.L. (1996), "Searching for Common Threads: Understanding the Multiple Effects of Diversity in Organizational Groups", Academy of Management Review, 21(2): 402-433.

Mutabazi, E et Pierre, $\mathrm{P}$, Pour un management interculturel De la diversité à la reconnaissance en entreprise, Paris, L'Harmattan, 2008.

Point, S, « La Charte de la Diversité : regards sur le discours des entreprises signataires », Management et Avenir, 2006, no 8, pp61-85.

Sabeg, Y, et Charlotin, C, La diversité dans l'entreprise : Comment la réaliser ? Paris, Editions d'Organisation, 2006.

Schnapper, D, Qu'est-ce que l'intégration ? Paris, folio, Gallimard, 2007.

Shapiro, D.L., Furst, S.A., Spreitzer, G.M. and Von Glinow, M.A. (2002), "Transnational Teams in the Electronic Age: Are Team Identity and High Performance at Risk?", Journal of Organizational Behavior, 23(4): 455-468.

Todd, E, L'enfance du monde, Structures familiales et développement, Paris, Seuil, 1984. Tyler, T.R. (1999), "Why People Cooperate with Organizations : an Identity-Based Perspective", Research in organizational behavior, 21: 241-246.

Watson, W.E., Kumar, K. and Michaelsen, L.K. (1993), “Cultural Diversity's Impact on Interaction Process and Performance: Comparing Homogeneous and Diverse Task Groups", Academy of Management Journal, 36(3): 590-602.

Weil, P, La République et sa diversité, Paris, Seuil, 2005. 